\title{
SOFT-MODE VS. LOCALIZED MOMENTS REGIMES OF SPIN FLUCTUATIONS IN ITINERANT ELECTRON MAGNETISM
}

\author{
A. Solontsov \\ State Center for Condensed Matter Physics \\ Rogova str. 5, 123060 Moscow, Russia. \\ and
}

Theoretical Laboratory, A.A. Bochvar Institute, 123060 Moscow, Russia

C. LACROIX

Laboratoire Louis Néel, C.N.R.S., 38042 Grenoble-Cedex, France

AND D. WAGNER

Teoretische Physik III, Ruhr-Universität Bochum 44780, Bochum, Germany

An analysis of different regimes of spin fluctuation behaviour in anharmonic itinerant electron magnets is presented basing on the recently worked out soft-mode theory of spin fluctuations.

PACS numbers: 75.10.Lp, 75.40.Gb, 75.50.Ee

Existing experimental data in metals present direct evidence for importance of overdamped spin fluctuation (SF) with frequencies softening near some points of the Brillouin zone related to magnetic instabilities. The SF behaviour depends on the mode-mode coupling, or spin anharmonicity, and may be separated into several well-defined regimes. The Fermi liquid (FL) regime of SF arising in the low-temperature limit changes to the soft-mode (SM) one at elevated temperatures and then to the localized moments (LM) regime characterized by the Curie-Weiss susceptibility and saturated local moments. As we show here in all regimes an important role is played by quantum zero-point SF which may give rise to strong spin anharmonicity. Ilowever, a meaningful classification of SF regimes as well as an analysis of spin anharmonicity is still lacking. A conventional description of SF effects is usually based on the mode-mode coupling [1], or self-consistent renormalization (SCR) [2] theory being valid only in the limit of weak spin anharmonicity [3]. Another approach is based on the constraint that the total local moment including the zero-point and thermal SF contributions is a conserved 
quantity (the "sum rule") [4]. This approach was shown to be valid in the opposite limit of strong SF coupling [3]. To account for spin anharmonicity beyond these limits a SM theory of SF was recently worked out basing on a variational procedure [3]. Here we use the SM theory to analyse different regimes of the SF behaviour in itinerant electron magnets.

The SF spectrum in the low frequency $\omega<\omega_{\mathrm{C}}$ regime is characterized by the dynamical magnetic susceptibility $\chi(\omega, k)$ taken usually in the form [2] $\chi^{-1}(\omega, k)=\chi^{-1}(k)-\mathrm{i} \omega / \Gamma(k)$, which defines the characteristic SF frequency $\omega_{\mathrm{SF}}(k)=\Gamma(k) \chi^{-1}(k)$ softening near $k=0$. IIere $\Gamma(k)=\Gamma_{0} k^{z-2}$ is the relaxation rate, $z$ is the dynamical exponent being equal to 2 for antiferro- and 3 for ferromagnetic fluctuations, $\chi^{-1}(k)=\chi^{-1}+c k^{2}$ is the static susceptibility, the wave vector $k\left(k<k_{\mathrm{C}}\right)$ is measured from a wave vector describing an instability, $\omega_{\mathrm{C}}(k)$ and $k_{\mathrm{C}}$ are the frequency and cut-off wave vector.

Comparing $\omega_{\mathrm{SF}}(k)$ with the thermal energy $k_{\mathrm{B}} T$ one can define the characteristic wave vector $k_{\mathrm{T}}$ of thermal SF, which is given by $k_{\mathrm{T}}=k_{\mathrm{C}}$ in the classical high-temperature limit, $T>T_{\mathrm{m}}$, and $k_{\mathrm{T}}=k_{\mathrm{C}}\left(T / T_{\mathrm{m}}\right)^{1 / z}$ in the low-temperature quantum regime, $T<T_{\mathrm{m}}$, where $T_{\mathrm{m}} \approx \hbar \Gamma_{0} c k_{\mathrm{C}}{ }^{z} / \alpha_{0} k_{\mathrm{B}}$ is the SF temperature, $\alpha_{0} \approx 1$ is a dimensionless parameter. Now it is possible to introduce a natural separation of the SF behaviour into three main regimes: (i) the FL one, arising in the low-temperature limit $c k_{T}^{2} \ll \chi_{0}^{-1} \approx \operatorname{const}(T)$, (ii) the SM regime, taking place near an instability, $\chi_{0}^{-1}(T) \ll c k_{T}^{2}$, and (iii) the LM regime arising at higher temperatures, $\chi_{0}^{-1}(T) \gg c k_{\mathrm{C}}^{2}$. The low-temperature regime is dominated by relatively high-frequency SF, $\hbar \omega_{\mathrm{SF}}(k) \gg k_{\mathrm{B}} T$, giving rise to the conventional FL behaviour. On the other hand, the SM regime is governed by thermally excited low-frequency $\mathrm{SF}, \mathrm{l} \omega_{\mathrm{SF}}(k) \leq k_{\mathrm{B}} T$. The $\mathrm{LM}$ regime is characterized by almost dispersionless SF, local in real space, which allows to describe them in terms of LM. In the intermediate temperature range $c k_{\mathrm{T}}^{2} \leq \chi_{0}^{-1}(T) \leq c k_{\mathrm{C}}^{2}$ a crossover from SM to LM regimes takes place.

IIere we focus on magnets close to a magnetic instability assuming a major role of strongly coupled SF. To describe the mode-mode coupling of SF we use a dimensionless spin anharmonicity parameter [3]

$$
g_{\mathrm{SF}}=\frac{\gamma_{0}}{3}\left|\frac{\partial\left(M_{\mathrm{L}}^{2}\right)}{\partial\left(\chi^{-1}\right)}\right|=g_{\mathrm{T}}+g_{\mathrm{Z} . \mathrm{P} .},
$$

which is a measure of the stiffness of averaged squared amplitude of SF, or squared local moments,

$$
M_{\mathrm{L}}^{2}=12 \hbar \sum_{k} \sum_{\omega} \chi(k) \frac{\omega \omega_{\mathrm{SF}}(k)}{\omega_{\mathrm{SF}}^{2}(k)+\omega^{2}}\left(N_{\omega}+\frac{1}{2}\right)=\left(M_{\mathrm{L}}^{2}\right)_{T}+\left(M_{\mathrm{L}}^{2}\right)_{Z . P .} .
$$

Here $\gamma_{0}$ is the coefficient in the $M^{4} / 4$ term of the Stoner free energy as a function of the magnetic order parameter $M, \sum_{\omega}=\int_{0}^{\infty} \mathrm{d} \omega / 2 \pi$, the factors $N_{\omega}=$ $\left[\exp \left(\hbar \omega / k_{\mathrm{B}} T\right)-1\right]^{-1}$ and $1 / 2$ define the thermal $\left(M_{\mathrm{L}}^{2}\right)_{\mathrm{T}}$ and zero-point $\left(M_{\mathrm{L}}^{2}\right)_{\text {z.P. }}$. contributions to $M_{\mathrm{L}}^{2}$, which also provides to distinguish the thermal $g_{\mathrm{T}}$ and zeropoint $g_{\text {Z.P. }}$ terms contributing to spin anharmonicity. 
First, we discuss temperature dependencies of thermal contributions to $M_{\mathrm{L}}^{2}$ and $g_{\mathrm{SF}}$ where the latter is given by the following interpolation formula:

$$
g_{\mathrm{T}} \sim \frac{\gamma_{0}\left(M_{\mathrm{L}}^{2}\right)_{\mathrm{T}}}{\sqrt{\chi^{-1}\left(\chi^{-1}+c k_{\mathrm{T}}^{2}\right)}} .
$$

As it follows from Eqs. (2) and (3) in the SM regime $\left(M_{\mathrm{L}}{ }^{2}\right)_{\mathrm{T}}=k_{\mathrm{B}} T k_{\mathrm{T}} / 2 \pi^{2} c$ increases $\sim T^{(1+z) / z}$ and gives rise to anharmonicity of thermal SF which is small except for the vicinity of the critical temperature $T_{\mathrm{C}}$, where $g_{\mathrm{T}} \sim \sqrt{T_{\mathrm{C}} \tau_{\mathrm{G}} /\left|T-T_{\mathrm{C}}\right|}$ is defined by the Ginzburg parameter $\tau_{\mathrm{G}}$, and diverges. In the $L M$ regime, provided $k_{\mathrm{B}} T \ll \hbar \omega_{\mathrm{C}}\left(k_{\mathrm{C}}\right) \sim k_{\mathrm{B}} T_{\mathrm{m}}$, Eq. (2) yiclds

$$
\left(M_{\mathrm{L}}^{2}\right)_{T}=3 k_{\mathrm{B}} T N_{0} \chi_{0}(T) G(T) \text {, }
$$

where the factor $G=\left(1 / N_{0}\right) \sum_{k} 2 z[\ln z-1 / 2 z-\Psi(z)] \leq 1$ accounts for the quantum reduction of the phase volume of thermal SF, $\Psi(z)$ is Euler's psi function, $z=\hbar \Gamma(k) / 2 \pi k_{\mathrm{B}} T \chi_{0}(T)$, and $N_{0}=k_{\mathrm{C}}^{3} / 6 \pi^{2}$ is the volume of the Brillouin zone. In the high-temperature limit $T \gg T_{\mathrm{m}}$ quantum effects are negligible, and one should set $G \rightarrow 1$ in Eq. (4). Below we assume that in the LM regime $\chi_{0}(T)$ has the Curie-Weiss form, which according to Eqs. (3) and (4) leads to a saturation of local moments, $\left(M_{\mathrm{L}}^{2}\right)_{\mathrm{T}}=$ const $(T)$, and to vanishing of spin anharmonicity $g_{\mathrm{T}} \sim 1 / T$.

Both in the SM and LM regimes spin anharmonicity due to thermal SF is small except for the critical region and one may regard this as a justification of perturbative approaches $[1,2]$ based on a weak mode-mode coupling constraint. Ilowever, this is not the case because of zero-point $\mathrm{SF}$ which may give rise to strong spin anharmonicity. In the SM regime from Eqs. (1) and (2) we have

$$
\begin{aligned}
& \left(M^{2}\right)_{\text {Z.P. }} \approx \hbar \Gamma\left(k_{\mathrm{C}}\right) N_{0} \alpha_{1}=M_{\mathrm{L} 0}^{2}, \\
& g_{\text {Z.P. }} \approx \frac{\hbar \gamma_{0} \Gamma\left(k_{\mathrm{C}}\right) N_{0}}{c k_{\mathrm{C}}{ }^{2}} \alpha_{2}=g_{0},
\end{aligned}
$$

where $\alpha_{1,2} \approx 1$ are dimensionless coeflicients. E.g., for a ferromagnetic instability setting $\omega_{\mathrm{C}}(k)=k v_{\mathrm{F}}$ (where $v_{\mathrm{F}}$ is the Fermi velocity) one gets $\alpha_{2}=(1 / 2 \pi) f$ $x \tan ^{-1}(1 / f)$, where $f=v_{\mathrm{F}} / \Gamma_{\mathrm{O}} c k_{\mathrm{C}}^{2}$. For typical itinerant magnets estimates yield $\left(M_{\mathrm{L}}^{2}\right)_{\text {z.P. }} \sim 1 \mu_{\mathrm{B}}^{2}$ and $g_{0} \sim 1$ (see Table). In the LM regime setting $T \rightarrow \infty$ we find that both $\left(M_{\mathrm{L}}^{2}\right)_{\text {Z.P. }} \sim T^{-2}$ and $g_{\text {Z.P. }} \sim T^{-3}$ vanish due to a reduction of quantum effects in the high-temperature limit.

\section{TABLE}

Quantum soft-mode $S F$ in low- $T_{\mathrm{C}}$ magnets.

\begin{tabular}{c|c|c|c|c}
\hline & $g_{0}$ & $T_{\mathrm{C}}[\mathrm{K}]$ & $T_{\mathrm{m}} / T_{\mathrm{C}}$ & $T_{\mathrm{L}} / T_{\mathrm{C}}$ \\
\hline $\mathrm{MnSi}$ & 3.7 & 29 & 7.3 & 1.4 \\
$\mathrm{Ni}_{3} \mathrm{Al}$ & 1.1 & 41 & 98 & 21 \\
$\mathrm{ZrZn}_{2}$ & 0.32 & 28 & 130 & 30
\end{tabular}

To account for effects of strong spin anharmonicity we recently worked out the SM theory of SF [3] going beyond a perturbative scheme. Basing on a variational procedure for the free energy with account of both zero-point and thermal 
SF, a magnet was shown to behave as an ensemble of thermally excited SF which are strongly renormalized with respect to zero-point effects. In the SM regime the temperature dependent susceptibility was related to the thermally induced local moments [3],

$$
\chi_{0}^{-1}(T)=\chi^{-1}(0)+\gamma\left(M_{\mathrm{L}}^{2}\right)_{\mathrm{T}}
$$

with the coupling constant

$$
\gamma=\gamma_{0} \frac{1-5 g}{1+6 g} \leq \gamma_{0}
$$

strongly influenced by zero-point SF, where $g=g_{0} \gamma / \gamma_{0}$ is the renormalized spin anliarmonicity parameter. The squared local moments are strongly temperature dependent,

$$
M_{\mathrm{L}}^{2}=(1-5 g)\left(M_{\mathrm{L}}^{2}\right)_{\mathrm{T}}+M_{\mathrm{L} 0}^{2},
$$

which gives rise to a magnetovolume effect proportional to $\left(M_{\mathrm{L}}^{2}\right)_{\mathrm{T}}$.

In the limit of weak anharmonicity, $g \approx g_{0} \ll 1$, the SM theory reduces to the conventional SCR approach [1,2]. In the opposite limit when $g_{0} \gg 1$ the squared moments are almost conserved, $M_{\mathrm{L}}^{2} \approx \operatorname{const}(T), \gamma \approx \gamma_{0} / 5 g_{0}$ and (7) yields the result of the "sum rule" approach [4]. Thus the SM theory generalizes the SCR and "sum rule" approaches and describes SF effects in the SM regime for arbitrary spin anharmonicity. The theory [3] holds up to a temperature $T_{\mathrm{L}}$, defined by $\chi_{0}^{-1}(T) \approx c k_{T}^{2}$, when a crossover to the LM regime takes place. For low- $T_{\mathrm{C}}$ ferromagnets an estimate yields $T_{\mathrm{L}} \approx\left(\alpha_{0} \alpha_{2} / 10 \pi g\right)^{3 / 2} T_{\mathrm{m}}$ where $\alpha_{0} \approx 1.897$.

In Table we present the values of the anharmonicity parameter $g_{0}$ taken from [3] and temperatures $T_{\mathrm{m}}$ and $T_{\mathrm{L}}$ characterizing quantum soft $\mathrm{SF}$ in low- $T_{\mathrm{C}}$ itinerant magnets $\mathrm{MnSi}, \mathrm{Ni}_{3} \mathrm{Al}$, and $\mathrm{ZrZn}_{2}$. As one can see from Table in all these magnets effects of spin anharmonicity due to zero-point $\mathrm{SF}$ are strong being the largest for $\mathrm{MnSi}$. In $\mathrm{Ni}_{3} \mathrm{Al}$ and $\mathrm{ZrZn} \mathrm{n}_{2}$ the SM regime holds up to rather high temperatures, $21 T_{\mathrm{C}}$ and $30 T_{\mathrm{C}}$, respectively, whereas in $\mathrm{MnSi}$ a crossover to the $\mathrm{LM}$ regime takes place almost near $T_{\mathrm{C}}$. In all magnets the SF temperature $T_{\mathrm{m}} \gg T_{\mathrm{C}}$ is rather high implying a quantum character of $\mathrm{SF}$. In MnSi it is one order of magnitude smaller, which probably gives rise to an almost heavy fermion behaviour of this compound.

\section{Acknowledgments}

The authors acknowledge partial support of Université Joseph Fourier, Russian Foundation for Basic Research, and Bundesministerium für Forschung und Technologie of Germany.

\section{References}

[1] K.K. Murata, S. Doniach, Phys. Rev. Lelt. 29, 285 (1972).

[2] T. Moriya, Spin Fluctuations in Itinerant Electron Magnetism, Springer, Berlin 1985.

[3] A. Solontsov, D. Wagner, Phys. Rev. B 51, 12410 (1995); J. Phys., Condens. Malter 6, 7395 (1994).

[4] T. Moriya, T. Takimoto, J. Phys. Soc. Jap. 64, 960 (1995). 